\title{
Transforming type 1 diabetes: the next wave of innovation
}

\author{
Daniel J. Drucker ${ }^{1}$
}

Received: 1 December 2020 / Accepted: 22 December 2020 / Published online: 6 February 2021

(C) The Author(s), under exclusive licence to Springer-Verlag GmbH, DE part of Springer Nature 2021

\begin{abstract}
The discovery of insulin in 1921 enabled pharmaceutical production of animal insulins for the treatment of people with type 1 diabetes by 1922. The last several decades have witnessed enormous scientific progress in the therapy of type 1 diabetes, yet some developments have been incremental, and insulin is not a cure. Herein, I highlight key scientific advances potentially poised to improve the quality of life and treatment outcomes in type 1 diabetes. These innovations range from newer insulin analogues to the development of smart insulins, oral and weekly insulins, glucose sensors and closed-loop insulin-delivery devices, as well as strategies for durable human beta cell replacement coupled with selective immune manipulation to preserve beta cell function. Finally, progress in the prediction and prevention of type 1 diabetes highlights the ongoing challenges and potential for altering the natural history of the disease or eliminating type 1 diabetes altogether.
\end{abstract}

Keywords Autoimmunity $\cdot$ Glucose $\cdot$ Immunology $\cdot$ Insulin $\cdot$ Pancreas $\cdot$ Pumps $\cdot$ Review $\cdot$ Stem cells

$\begin{array}{ll}\text { Abbreviations } \\ \text { GLP-1 } & \text { Glucagon-like peptide-1 } \\ \text { I338 } & \text { Insulin } 338 \\ \text { SGLT2i } & \text { Sodium-glucose cotransporter } 2 \text { inhibitors } \\ \text { Treg } & \text { Regulatory T cell }\end{array}$

\section{Introduction}

In this issue of Diabetologia, a series of papers linked to the scientific celebration of the 100th anniversary of the discovery of insulin at the University of Toronto, ON, Canada (insulin100.com) describe important advances in the science of type 1 diabetes, while highlighting challenges and barriers to be surmounted. Herein, I summarise key areas of innovation that show great promise for transforming diabetes care and improving the lives of people with type 1 diabetes (Fig. 1).

Daniel J. Drucker drucker@lunenfeld.ca

1 Department of Medicine, Lunenfeld-Tanenbaum Research Institute, Mount Sinai Hospital, University of Toronto, Toronto, ON, Canada

\section{Newer insulins}

After more than 60 years of producing animal insulins for the treatment of type 1 diabetes, the introduction of recombinant human insulin almost four decades ago has spurred the development and formulation of rapid-acting insulin analogues, as well as newer basal insulins, such as insulin degludec together with more concentrated U300 insulin glargine. Many rapidacting analogues are now deployed within insulin pump regimens, allowing for more rapid onset of action and faster dissipation of post-meal-related insulin action. The newer longacting basal insulins achieve more effective $24 \mathrm{~h}$ insulinisation in the majority of patients, while reducing rates of nocturnal hypoglycaemia. Flexibility in the time of dosing of insulin degludec may also be useful for some individuals with type 1 or type 2 diabetes [1]. Whether switching to newer longacting basal insulins confers meaningful sustained benefits for people with type 1 diabetes is unknown.

\section{New methods for delivery of insulin}

Once-weekly insulin An investigational once-weekly formulation of an acylated degradation-resistant insulin, at present provisionally designated insulin icodec, was assessed in a 26 week Phase II trial in people with type 2 diabetes. Compared with once-daily insulin glargine U100, starting at a dose of $70 \mathrm{U}$ and up-titrated weekly to a mean dose of 
Fig. 1 Strategies for improving therapeutic outcomes in people with type 1 diabetes. The various technologies and biological strategies under development for the treatment of type 1 diabetes and its complications are shown. This figure is available as a downloadable slide

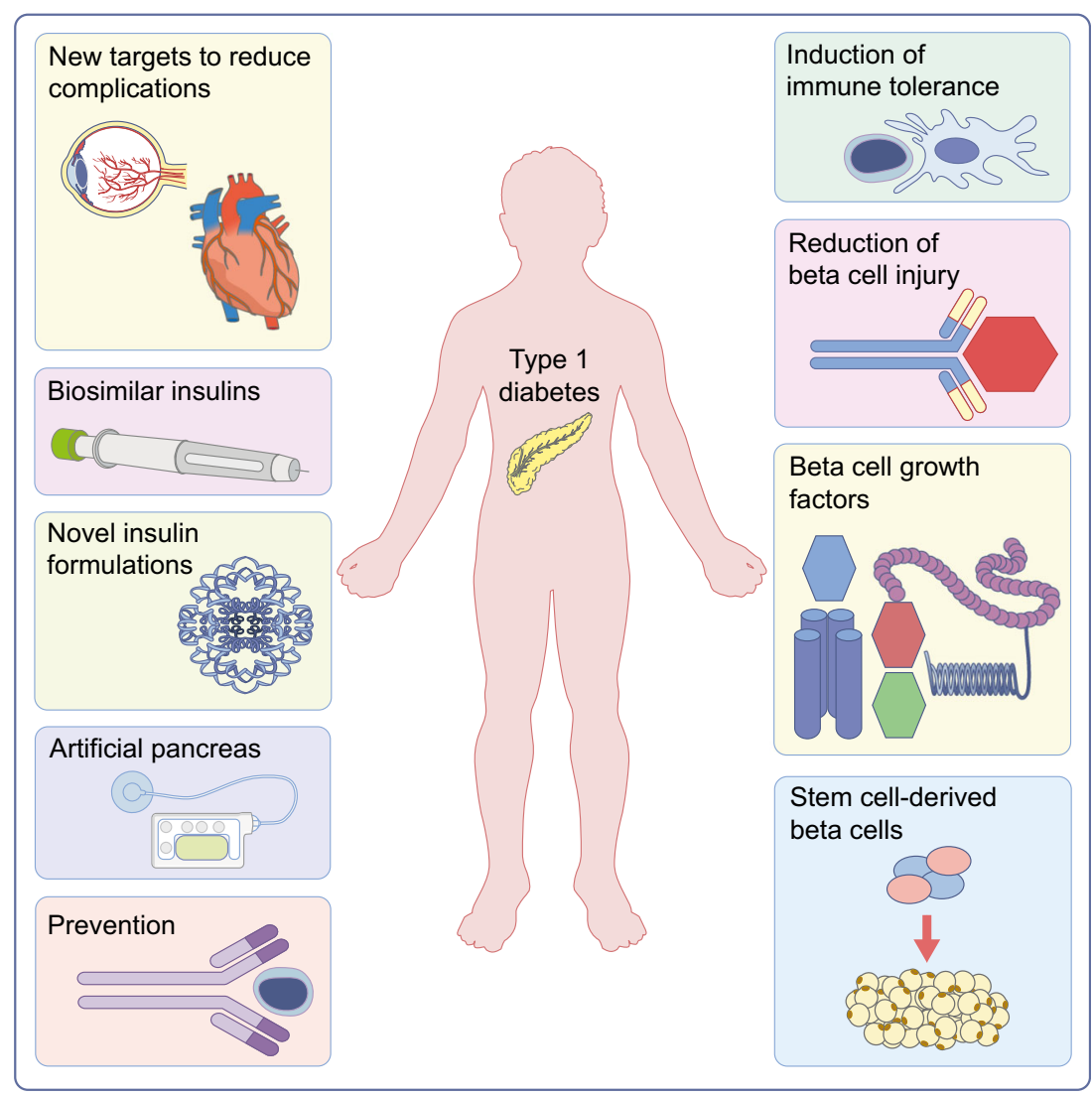

$241 \mathrm{U} /$ week, insulin icodec exhibited a comparable safety and efficacy profile when added to a background regimen of metformin with or without a dipeptidyl peptidase-4 inhibitor [2]. Rates of minor (level 1) hypoglycaemia and the relative reduction of $\mathrm{HbA}_{1 \mathrm{c}}$ was numerically greater with insulin icodec than insulin glargine U100. Reducing the frequency of basal injections from 365 to 52 per year is appealing. Whether once-weekly basal insulins will provide substantial advantages while maintaining an excellent safety and efficacy profile in people with type 1 diabetes will require considerable study. Having a substantial reservoir of basal insulin on board has implications for management of unexpected intercurrent illness, vigorous exercise, and nocturnal or new-onset hypoglycaemia; these areas require additional investigation in the context of type 1 diabetes.

Oral insulin The feasibility of oral insulin administration, viewed by some as an important alternative to enable reduction of the number of injections, has been extensively investigated for decades in preclinical studies and, more recently, in people with type 1 and type 2 diabetes. While results of shortterm animal studies assessing oral insulin frequently show acute reductions in blood glucose, clinical development efforts are challenged by inter-individual variability in gastric emptying and pharmacokinetics and very low bioavailability [3]. Both short-acting and long-acting oral insulins are being studied. The efficacy and safety of insulin 338 (I338), a longacting acylated insulin analogue co-formulated with sodium caprate for once-daily oral administration, was assessed in an 8 week trial in insulin-naive people with type 2 diabetes treated with oral glucose-lowering medicines. No difference in the magnitude of glucose reduction or rates of adverse events was detected in people randomised to I338 vs insulin glargine [4]. Nevertheless, the clinical development programme for I338 was discontinued owing to the high doses required (58-fold higher than insulin glargine) to achieve acceptable pharmacokinetics and the questionable commercial feasibility of marketing a new, more expensive insulin product. Substantial numbers of people with type 1 diabetes continue to be treated using regimens encompassing once-daily basal insulin administration. Hence, the feasibility of a longer acting basal insulin remains theoretically attractive. However, costeffective development will likely require additional progress in achieving greater bioavailability, alongside careful ongoing study of patients taking oral insulins.

The possibility of developing rapid-acting oral insulin formulations for the treatment of type 1 diabetes continues to be explored. ORMD-0801 is an oral insulin co-formulated with enzyme inhibitors that is currently being assessed for the treatment of type 1 and type 2 diabetes [3]. The successful development of rapid-acting oral insulin preparations that are effective in a wide variety of people with type 1 diabetes 
remains challenging. Given inter- and intra-individual variability in gastric emptying and absorption of peptides, together with a need for reproducible pharmacokinetics and rapid onset commensurate with meal ingestion, it seems unlikely that oral prandial rapid-acting insulins will be equivalent in efficacy and safety in people with type 1 diabetes when compared with newer rapid-acting injectable insulin analogues.

Miscellaneous non-injectable insulins An inhaled rapid-acting insulin has been approved for diabetes therapy; however, its ultimate place in the type 1 diabetes treatment paradigm remains uncertain. Inhaled insulin promises a more rapid rate of onset and a shorter duration of action, more closely mimicking secretion of postprandial insulin and, ideally, reducing rates of hypoglycaemia [5].

Other innovations in the delivery of insulin have included the development of subcutaneous patches, orally ingested unfolding microneedle devices, and ingestible microapplicators that adhere to the gastrointestinal tissue and deliver calibrated doses of insulin in preclinical studies $[3,6]$. An ultimate goal of these technologies is to remove the need to deliver insulin by injection multiple times daily, while maintaining effective $24 \mathrm{~h}$ /day insulinisation.

\section{Smart glucose-sensitive insulins}

Towards the objective of reducing insulin injections while minimising hypoglycaemia, there is intense interest in the development of effective new 'smart' or glucose-sensitive insulins that theoretically facilitate intensification of insulin therapy in a safe manner. Ideally, insulin could be administered and sequestered within an inactive reservoir, such as in a patch or a biological or mechanical delivery device, and released as needed via exquisitely glucose-sensitive mechanisms. Multiple new molecular entities are being explored, incorporating glucose-sensitive modifications within the insulin molecule or within linked carriers or fusion proteins. These formulations may include lectins, gels and polymers, which are designed to release insulin or switch insulin to a bioactive conformation in proportion to gradients in hyperglycaemia [7]. Ideally, a glucose excursion within the hyperglycaemic range would lead to rapid release of a precise quantity of bioactive insulin, with the release or active-conformation switch mechanism being terminated equally rapidly upon return to normoglycaemia. Substantial progress has been made with regard to technologies enabling glucose-sensitive insulin delivery; however, the risks of overshooting or underdelivering the precise quantities of insulin required needs careful scrutiny during development and in clinical trials [7].

Major challenges and unresolved questions surround the development and optimisation of novel glucose-sensitive insulins for management of type 1 diabetes. For example, should glucose-sensitive insulins be preferentially developed as adjunctive basal or short-acting preparations, or simply as universally acting insulins stored in compartments that will be continuously available for fine-tuning insulin release in relation to ambient glucose? Will the on/off mechanism be sufficiently rapid and robust to manage acute swings in blood glucose levels that may occur during repeated bouts of intense exercise or during rapid development of inadvertent hypoglycaemia? How will adequate basal insulinisation and avoidance of ketoacidosis be achieved during a state of prolonged fasting or in settings of intercurrent illness characterised by relative euglycaemia? Will a glucosesensitive insulin-delivery mechanism provide sufficient basal insulin release to engage receptors in key organs, such as the liver, brain and adipose tissue, in the fasting or prolonged euglycaemic state? Answering these questions will require careful experimental scrutiny in preclinical studies and, subsequently, in clinical trials.

\section{Innovation in insulin-delivery systems: towards the artificial pancreas}

Progress in partially or fully 'closing the loop' has been rapid and impressive, with continuous improvement in the development and validation of devices that couple continuous glucose sensing to partially or fully automated control of insulin secretion. This has been reviewed in this issue by Boughton and Hovorka [8] and Jarosinski et al. [7]. Continuous glucosesensing and sensor-augmented insulin-delivery devices have evolved rapidly and are increasingly being adopted in many worldwide regions for the treatment of type 1 diabetes. Rapid development of glucose-sensing and insulin-delivery technologies, together with 'suspend-before-low' technology and predictive algorithms, will enable improved hybrid and fully automated closed-loop devices to evolve. However, the widespread uptake of these devices depends on further validation in multiple populations and real-world clinical scenarios, including intensive exercise. Considerable progress has been made in the development of a more fully automated artificial pancreas, coupling glucose sensing to insulin delivery, and the feasibility of simultaneously delivering low-dose glucagon is also being explored. Uploading data to the cloud, coupled with artificial intelligence-enabled algorithms for data analysis and insulin dose recommendations, may provide a useful adjunct to self-management, ideally enhancing the traditional interactions between healthcare providers and people with type 1 diabetes. Greater miniaturisation of these delivery systems and exploration of the feasibility of smarter closedloop devices, with longer duration of use (i.e. time the device can be implanted and left alone without a new supply of insulin or new batteries etc), designed for intraperitoneal 
implantation with refillable insulin options, may herald improved possibilities for some. Dual-hormone delivery systems combining glucagon or amylin with insulin are also under investigation, promising a lower risk of hypoglycaemia and greater time in range [8]. Excitingly, under the mantra of 'we are not waiting', parallel innovation in open-source do-ityourself algorithms has further advanced therapeutic options for automated insulin delivery in some people with type 1 diabetes. With advances in manufacturing, one anticipates that the price of these devices, which is currently a barrier to adoption for many, will come down, facilitating greater uptake and use in more populations.

\section{Hypoglycaemia}

As highlighted by David Nathan in this issue [9], intensive insulin therapy in the Diabetes Control and Complications Trial was associated with a threefold increased risk in hypoglycaemia. The use of newer rapid-acting analogues and long-acting basal insulins, together with continuous glucose monitoring, has reduced the rates of hypoglycaemia in many people with type 1 diabetes. However, despite these important advances, the consequences of hypoglycaemia remain burdensome and represent a substantial challenge and barrier to intensification of glycaemic control in many people with type 1 diabetes [10]. A great deal of progress has been made in understanding the mechanisms of glucose sensing in the brain and peripheral tissues, identifying how multiple defects in these physiological mechanisms contribute to hypoglycaemia susceptibility in people with type 1 diabetes. It remains unclear if therapeutic approaches to restore glucose sensing and improve defective counterregulation are feasible. Whether co-administration of low-dose glucagon (together with insulin) in pumps to prevent hypoglycaemia will prove clinically useful is a question that requires ongoing research.

\section{Cell regeneration and replacement therapies}

Notwithstanding exciting advances in the pharmacokinetics of new insulin molecules and innovative insulin-delivery systems, a long-standing goal is to eliminate the daily need to manage insulin delivery. The field has steadily evolved from pancreas and islet transplantation towards efforts directed at beta cell regeneration and implantation of stem cellderived beta cells. Ideally, safe, effective and affordable beta cell replacement therapies will be developed that can be administered without lifelong broad immunosuppression.

There continues to be enormous interest in the regeneration of functional beta cell mass to achieve therapeutic benefit in people with type 1 diabetes. These efforts are based on substantial evidence that C-peptide may remain detectable for decades after diagnosis and that some individuals have populations of residual pancreatic beta cells for prolonged periods of time after the onset of type 1 diabetes [11]. In this regard, future advances in the imaging of functional human beta cells in situ would prove useful for correlating changes in beta cell mass with concomitant evaluation of beta cell secretion. Whether sufficiently robust and selective regeneration of endogenous residual beta cells (coupled with immunosuppression) is feasible remains unclear. Advances in the identification of human beta cell growth factors support the possibility of achieving expansion of human beta cells prior to transplantation ex vivo, perhaps in combination with agents such as glucagon-like peptide-1 (GLP-1) receptor agonists. Alternatively, these regenerative combinations might be administered in vivo for brief intermittent intervals to increase marginal beta cell mass [12].

For many, the holy grail of type 1 diabetes research remains cell replacement therapy, an area that has witnessed exciting progress, as outlined by Douglas Melton in this review series [13]. Normal human beta cells function within a complex topologically organised multicellular islet environment, with beta cell function constantly being fine-tuned by local paracrine, intercellular, neural and vascular feedback. The extent to which stem cell-derived human beta cells can be coaxed to function normally for prolonged periods of time in a new environment that only partially mimics the normal organisation of pancreatic islets requires ongoing scrutiny.

The islet replacement field has simultaneously pursued innovative approaches for shielding new implanted beta cells from the immune system through the use of materials that enable vascularisation and oxygenation while excluding immune cells and antibodies. Ongoing molecular approaches include the development of strategies to block or reverse autoimmunity and induce allotolerance, thereby permitting transplant engraftment and durable function without the need for continuous immunosuppression. This might be achieved by genetic elimination of key molecular targets in new human beta cells that are recognised by the immune system in people with type 1 diabetes, coupled with highly selective targeted manipulation of the immune system to precisely disable immune effectors responsible for beta cell destruction [14]. Abolishing molecular targets of the autoimmune attack in differentiated stem cell-derived human beta cells appears feasible, yet may inadvertently activate different components of the immune system, while also potentially posing a risk for tumour formation. Multiple approaches to achieving immune tolerance of newly generated human beta cells are being explored and rapid progress in this exciting field is expected. Moreover, genome editing and engineering strategies have evolved to couple detection of cell proliferation with induction of cell death, providing built-in mechanisms for eliminating rogue cells with tumourigenic potential [15]. 


\section{Complementary strategies for innovation in type 1 diabetes}

Many of the technologies discussed herein are highly innovative, pushing the boundaries of chemistry, engineering, immunology and cellular and molecular biology. Yet there remains ample room for exploring simple but potentially important innovation to improve the lives of people with type 1 diabetes. There is now substantial evidence supporting use of sodium - glucose cotransporter 2 inhibitors (SGLT2i) for the prevention of chronic kidney disease, heart failure and major cardiovascular events in people with type 2 diabetes. SGLT2i also provide an opportunity for intensification of glycaemic control without body-weight gain. Technical advances in noninvasive monitoring for ketonaemia may provide new opportunities for improving the safety of SGLT2i in people with type 1 diabetes, enabling relevant outcome studies. Similarly, GLP-1 receptor agonists reduce the rates of myocardial infarction, stroke and cardiovascular death in individuals with type 2 diabetes, likely, in part, through glucoseindependent mechanisms [16]. Although the pathophysiology of these complications may not be identical in type 1 vs type 2 diabetes, it seems reasonable to investigate the potential for these agents to safely reduce cardiorenal morbidity and mortality in individuals with type 1 diabetes at higher risk for these complications.

\section{Prevention of type 1 diabetes and preservation of beta cell function}

While exploring better options for intelligent insulin delivery, one must simultaneously strive to meet the challenge of preventing type 1 diabetes. Enormous progress in the identification of individuals at risk has made this goal more feasible. Intriguingly, longitudinal autoantibody screening programmes have demonstrated that a proportion of islet autoantibody-positive individuals may serorevert towards negative status, most often seen with insulin autoantibodies, potentially refining the timing and targeting of type 1 diabetes prevention interventions [17]. Serial changes in glucoseresponse curves following oral glucose challenge may further identify antibody-positive individuals at greater risk of progression to type 1 diabetes [18]. While autoantibody status has proven enormously valuable for type 1 diabetes prediction, the combination of autoantibody status, genetic risk score and family history was found to improve prediction of type 1 diabetes in high-risk children, allowing for further refinement of follow-up strategies and planned enrolment for intervention trials [19].

The clinical progression to type 1 diabetes was delayed in relatives of individuals with this disease (who are at high risk of developing type 1 diabetes) who were treated with the anti-
CD3 antibody teplizumab [20]. Efforts to prevent or slow the progressive deterioration of beta cell function with early immune interventions in children and young adults with newly diagnosed type 1 diabetes have also been met with success. Individuals who were administered golimumab, an anti-TNF- $\alpha$ monoclonal antibody, demonstrated better preservation of meal-stimulated insulin secretion after 52 weeks of therapy relative to control participants, with maintenance treatment infusions of golimumab being given every 2 weeks [21].

Complementary strategies are focusing on augmenting the numbers of regulatory $\mathrm{T}$ cells (Tregs) to restore immune tolerance using adoptive Treg transfer, perhaps using bioengineered antigen-specific Tregs, or a combination of cytokines or immunomodulatory agents to boost endogenous Treg populations [22]. The long-term safety of these innovative immunomodulatory therapies for suppression of the immune attack in susceptible individuals, or perhaps used in combination with beta cell replacement, will require carefully scrutiny in clinical trials.

Important questions surround the optimal dosing regimens and duration of immunosuppressive therapy required to produce the meaningful clinical benefit theoretically associated with the preservation of C-peptide. These benefits might include reduced rates of ketoacidosis, lower $\mathrm{HbA}_{1 \mathrm{c}}$, greater time in range, lower rates of hypoglycaemia and, ideally, reduced rates of complications beyond those achievable with conventional intensification of insulin therapy [10]. It seems likely that combinations of immunosuppressive agents would produce even greater preservation of beta cell function than one therapy alone; however, understanding the long-term safety of such approaches is not trivial.

\section{Access to innovation and future therapies for type 1 diabetes}

As we approach 100 years of insulin, scientific progress over the last four decades has been encouraging and has greatly improved the lives of people with type 1 diabetes. Advances in implementation science and the evaluation of the impact of each new technological advance will aid in judging real success from flashy, yet incremental innovation. However, this is no time for the scientific community to take a bow and briefly rest on its laurels. There continues to be a huge disparity in therapeutic options available to people with type 1 diabetes and tremendous region- and country-specific differences in diabetes-related outcomes. Despite the introduction of a slew of newer insulin analogues and the development of biosimilar insulins, the insulin supply chain remains highly fragmented and reliable global access to affordable insulin for people with type 1 diabetes remains uneven, even in some wealthy nations [23]. 
Notwithstanding the perceived value of innovative new insulins, there continues to be substantial debate about the pricing vs value delivered for innovation in type 1 diabetes care. As noted by Chan and colleagues, more than one million people were diagnosed with type 1 diabetes in 2017 and more than 14,000 people under the age of 25 years succumbed to the illness [24]. A substantial proportion of this mortality is likely preventable through delivery and application of standards of care that are widely available in some, but not all countries. Indeed, suboptimal care of people with type 1 diabetes produces tremendous individual hardship and impairs the economic health and productivity of nations [24]. In conjunction with the 100th anniversary of insulin, the World Health Organization has simultaneously launched the Global Diabetes Compact [25], with recommendations for actions enabling more affordable technology and insulins to be forthcoming in April 2021. As we contemplate the future of scientific innovation in type 1 diabetes, we must simultaneously ensure that new developments are affordable, costeffective, safe, easy to implement and designed to benefit large diverse populations with type 1 diabetes.

Until we can prevent diabetes, we need to learn how to more effectively minimise its impact and prevent its complications. The following words of Fredrick Banting [26] almost 100 years ago still resonate today:

\section{Insulin is not a cure for diabetes; it is a treatment. It enables the diabetic to burn sufficient carbohydrates, so that proteins and fats may be added to the diet in suffi- cient quantities to provide energy for the economic burdens of life.}

Frederick Banting

The gift of insulin transformed a previously fatal disorder into a chronic disease requiring intensive daily management. Although the science discussed herein represents a snapshot of major areas under investigation, the next major advance in type 1 diabetes may come, unexpectedly, from unconventional science or nascent technologies not yet fully mature. The power of modern science and the needs of people with type 1 diabetes demands our complete dedication to the development of newer transformational approaches directed at improving the management and eliminating the development of type 1 diabetes.

Supplementary Information The online version contains a slide of the figure for download available at https://doi.org/10.1007/s00125-02105396-5.

Funding DJD is supported by a Banting and Best Diabetes Centre-Novo Nordisk Chair in Incretin biology, the Novo Nordisk Foundation-Sinai Health Fund in Regulatory Peptides, and CIHR grant 154321.

Author's relationships and activities DJD has served as an advisor or consultant or speaker within the past 12 months to Boehringer Ingelheim
Inc., Forkhead Biotherapeutics, Intarcia Therapeutics, Kallyope, Merck Research Laboratories, Eli Lilly Inc. and Novo Nordisk Inc. Neither DJD nor his family members hold stock directly or indirectly in any of these companies. GLP-2 (not discussed in this review) is the subject of a patent license agreement between Shire Inc. and the University of Toronto, Toronto General Hospital (UHN) and DJD.

Contribution statement DJD is the sole author and takes full responsibility for the published work

\section{References}

1. Philis-Tsimikas A, Klonoff DC, Khunti K et al (2020) Risk of hypoglycaemia with insulin degludec versus insulin glargine U300 in insulin-treated patients with type 2 diabetes: the randomised, head-to-head CONCLUDE trial. Diabetologia 63(4): 698-710. https://doi.org/10.1007/s00125-019-05080-9

2. Rosenstock J, Bajaj HS, Janez A et al (2020) Once-weekly insulin for type 2 diabetes without previous insulin treatment. $\mathrm{N}$ Engl $\mathrm{J}$ Med 383(22):2107-2116. https://doi.org/10.1056/ NEJMoa2022474

3. Drucker DJ (2020) Advances in oral peptide therapeutics. Nat Rev Drug Discov 19(4):277-289. https://doi.org/10.1038/s41573-0190053-0

4. Halberg IB, Lyby K, Wassermann K, Heise T, Zijlstra E, PlumMorschel L (2019) Efficacy and safety of oral basal insulin versus subcutaneous insulin glargine in type 2 diabetes: a randomised, double-blind, phase 2 trial. Lancet Diabetes Endocrinol 7(3):179 188. https://doi.org/10.1016/S2213-8587(18)30372-3

5. Seaquist ER, Blonde L, McGill JB et al (2020) Hypoglycaemia is reduced with use of inhaled Technosphere $\AA$ insulin relative to insulin aspart in type 1 diabetes mellitus. Diabet Med 37(5):752-759. https://doi.org/10.1111/dme.14202

6. Abramson A, Caffarel-Salvador E, Khang M et al (2019) An ingestible self-orienting system for oral delivery of macromolecules. Science 363(6427):611-615. https://doi.org/10.1126/science. aau2277

7. Jarosinski MA, Dhayalan B, Rege N, Chatterjee D, Weiss MA (2021) 'Smart' insulin delivery technologies and intrinsic glucoseresponsive analogues. Diabetologia (in press)

8. Boughton CK, Hovorka R (2021) New closed-loop insulin systems. Diabetologia. https://doi.org/10.1007/s00125-021-05391-w

9. Nathan DM (2021) Realising the long-term promise of insulin therapy: the DCCT/EDIC study. Diabetologia. https://doi.org/10.1007/ s00125-021-05397-4

10. Amiel SA (2021) The consequences of hypoglycaemia. Diabetologia. https://doi.org/10.1007/s00125-020-05366-3

11. Keenan HA, Sun JK, Levine J et al (2010) Residual insulin production and pancreatic ss-cell turnover after 50 years of diabetes: Joslin Medalist Study. Diabetes 59(11):2846-2853. https://doi.org/10. 2337/db10-0676

12. Ackeifi C, Wang P, Karakose E et al (2020) GLP-1 receptor agonists synergize with DYRK1A inhibitors to potentiate functional human beta cell regeneration. Sci Transl Med 12(53):eaaw9996. https://doi.org/10.1126/scitranslmed.aaw9996

13. Melton D (2021) The promise of stem cell-derived islet replacement therapy. Diabetologia. https://doi.org/10.1007/s00125-020-053672

14. Bluestone JA, Tang Q (2020) Solving the puzzle of immune tolerance for beta-cell replacement therapy for type 1 diabetes. Cell Stem Cell 27(4):505-507. https://doi.org/10.1016/j.stem.2020.09. 008 
15. Liang Q, Monetti C, Shutova MV et al (2018) Linking a celldivision gene and a suicide gene to define and improve cell therapy safety. Nature 563(7733):701-704. https://doi.org/10.1038/ s41586-018-0733-7

16. McLean BA, Wong CK, Campbell JE, Hodson DJ, Trapp S, Drucker DJ (2020) Revisiting the complexity of GLP-1 action-from sites of synthesis to receptor activation. Endocr Rev. https://doi.org/ 10.1002/dev.22079

17. Pollanen PM, Ryhanen SJ, Toppari J et al (2020) Dynamics of islet autoantibodies during prospective follow-up from birth to age 15 years. J Clin Endocrinol Metab 105(12):e4638-e4651. https://doi. org/10.1210/clinem/dgaa624

18. Ismail HM, Cleves MA, Xu P et al (2020) The pathological evolution of glucose response curves during the progression to type 1 diabetes in the TrialNet Pathway to Prevention study. Diabetes Care 43(11):2668-2674. https://doi.org/10.2337/dc20-0701

19. Ferrat LA, Vehik K, Sharp SA et al (2020) A combined risk score enhances prediction of type 1 diabetes among susceptible children. Nat Med 26(8):1247-1255. https://doi.org/10.1038/s41591-0200930-4

20. Herold KC, Bundy BN, Long SA et al (2019) An anti-CD3 antibody, Teplizumab, in relatives at risk for type 1 diabetes. $\mathrm{N}$ Engl $\mathrm{J}$ Med 381(7):603-613. https://doi.org/10.1056/NEJMoa1902226
21. Quattrin T, Haller MJ, Steck AK et al (2020) Golimumab and Betacell function in youth with new-onset type 1 diabetes. N Engl J Med 383(21):2007-2017. https://doi.org/10.1056/NEJMoa2006136

22. Marfil-Garza BA, Hefler J, Bermudez De Leon M, Pawlick R, Dadheech N, Shapiro AMJ (2020) Progress in translational regulatory $\mathrm{T}$ cell therapies for type 1 diabetes and islet transplantation. Endocr Rev. https://doi.org/10.1210/endrev/bnaa028

23. Beran D, Lazo-Porras M, Mba CM, Mbanya JC (2021) A global perspective on the issue of access to insulin. Diabetologia. https:// doi.org/10.1007/s00125-020-05375-2

24. Chan JCN, Lim LL, Wareham NJ et al (2020) The Lancet commission on diabetes: using data to transform diabetes care and patient lives. Lancet 396(10267):2019-2082. https://doi.org/10.1016/ S0140-6736(20)32374-6

25. World Health Organization (2020). The WHO Global Diabetes Compact: uniting around a common agenda for diabetes. Available from: www.who.int/docs/default-source/world-diabetesday/global-diabetes-compact-final.pdf Accessed 13 January 2021

26. Banting FG (1925) Frederick G. Banting Nobel Lecture. Available from: www.nobelprize.org/prizes/medicine/1923/banting/lecture/. Accessed: 13 January 2021

Publisher's note Springer Nature remains neutral with regard to jurisdictional claims in published maps and institutional affiliations. 